\title{
A Dark Energy Model with Higher Order Derivatives of $H$ in the $f(R, T)$ Modified Gravity Model
}

\author{
Antonio Pasqua, ${ }^{1}$ Surajit Chattopadhyay, ${ }^{2}$ and Ratbay Myrzakulov ${ }^{3}$ \\ ${ }^{1}$ Department of Physics, University of Trieste, Via Valerio 2, 34127 Trieste, Italy \\ ${ }^{2}$ Pailan College of Management and Technology, Bengal Pailan Park, Kolkata 700 104, India \\ ${ }^{3}$ Eurasian International Center for Theoretical Physics, Eurasian National University, Astana 010008, Kazakhstan \\ Correspondence should be addressed to Surajit Chattopadhyay; surajit_2008@yahoo.co.in
}

Received 20 September 2013; Accepted 23 October 2013; Published 29 January 2014

Academic Editors: B. Bilki and C. A. D. S. Pires

Copyright (C) 2014 Antonio Pasqua et al. This is an open access article distributed under the Creative Commons Attribution License, which permits unrestricted use, distribution, and reproduction in any medium, provided the original work is properly cited.

\begin{abstract}
We consider a model of dark energy (DE) which contains three terms (one proportional to the squared Hubble parameter, one to the first derivative, and one to the second derivative with respect to the cosmic time of the Hubble parameter) in the light of the $f(R, T)=\mu R+\nu T$ modified gravity model, with $\mu$ and $\nu$ being two constant parameters. $R$ and $T$ represent the curvature and torsion scalars, respectively. We found that the Hubble parameter exhibits a decaying behavior until redshifts $z \approx-0.5$ (when it starts to increase) and the time derivative of the Hubble parameter goes from negative to positive values for different redshifts. The equation of state $(E O S)$ parameter of DE and the effective EoS parameter exhibit a transition from $\omega<-1$ to $\omega>-1$ (showing a quintom-like behavior). We also found that the model considered can attain the late-time accelerated phase of the universe. Using the statefinder parameters $r$ and $s$, we derived that the studied model can attain the $\Lambda$ CDM phase of the universe and can interpolate between dust and $\Lambda \mathrm{CDM}$ phase of the universe. Finally, studying the squared speed of sound $v_{s}^{2}$, we found that the considered model is classically stable in the earlier stage of the universe but classically unstable in the current stage.
\end{abstract}

\section{Introduction}

The late-time accelerated expansion of the universe (which is well-established from different cosmological observations) $[1,2]$ is a major challenge for cosmologists. The universe underwent two phases of accelerated expansion: the inflationary stage in the very early universe and a late-time acceleration in which our universe entered only recently. Models trying to explain this late-time acceleration are dubbed as dark energy (DE) models. An important step toward the comprehension of the nature of $\mathrm{DE}$ is to understand whether it is produced by a cosmological constant $\Lambda$ or it is originated from other sources dynamically changing with time [3]. For good reviews on DE see [4-6].

In a recent paper, Nojiri and Odintsov [7] described the reasons why modified gravity approach is extremely attractive in the applications for late accelerating universe and DE. Another good review on modified gravity was made by Clifton et al. [8]. Many different theories of modified gravity have been recently proposed: some of them are $f(R)$ (with $R$ being the Ricci scalar curvature) $[9,10], f(T)$ (with $T$ being the torsion scalar) [11-14], Hořava-Lifshitz [15, 16], and Gauss-Bonnet [17-20] theories.

In this paper, we concentrate on $f(R, T)$ gravity, with $f$ being in this case a function of both $R$ and $T$, manifesting a coupling between matter and geometry. Before going into the details of $f(R, T)$ gravity, we describe some important features of the $f(R)$ gravity. The recent motivation for studying $f(R)$ gravity came from the necessity to explain the apparent late-time accelerating expansion of the universe. Detailed reviews on $f(R)$ gravity can be found in [21-24]. Thermodynamic aspects of $f(R)$ gravity have been investigated in the works of $[25,26]$. A generalization of the $f(R)$ modified theory of gravity that includes an explicit coupling of an arbitrary function of $R$ with the matter Lagrangian density $L_{m}$ leads to a non-geodesic motion of massive particles and an extra force, orthogonal to the four-velocity, arises. [27]. Harko et al. [28] recently suggested an extension of standard general 
relativity, where the gravitational Lagrangian is given by an arbitrary function of $R$ and $T$ and called this model $f(R, T)$. The $f(R, T)$ model depends on a source term, representing the variation of the matter stress-energy tensor with respect to the metric. A general expression for this source term can be obtained as a function of the matter Lagrangian $L_{m}$. In a recent paper, Myrzakulov [29] proposed $f(R, T)$ gravity model and studied its main properties of FRW cosmology. Moreover, Myrzakulov [30] recently derived exact solutions for a specific $f(R, T)$ model which is a linear combination of $R$ and $T$, that is, $f(R, T)=\mu R+\nu T$, where $\mu$ and $\nu$ are two free constant parameters. Moreover, it was demonstrated that, for some specific values of $\mu$ and $\nu$, the expansion of universe results to be accelerated without the necessity to introduce extra dark components. Recently, Chattopadhyay [31] studied the properties of interacting Ricci DE considering the model $f(R, T)=\mu R+\nu T$. Pasqua et al. [32] recently considered the modified holographic Ricci dark energy (MHRDE) model in the context of the specific $f(R, T)$ model we are considering in this work. Moreover, Alvarenga et al. [33] studied the evolution of scalar cosmological perturbations in the metric formalism in the framework of $f(R, T)$ modified theory of gravity.

In this work, we consider a DE model proposed in the recent paper of Chen and Jing [34]. The DE model considered contains three different terms, one proportional to the squared Hubble parameter, one to the first derivative with respect to the cosmic time of the Hubble parameter, and one proportional to the second derivative with respect to the cosmic time of the Hubble parameter:

$$
\rho_{\mathrm{DE}}=\varepsilon \frac{\ddot{H}}{H}+\lambda \dot{H}+\theta H^{2},
$$

where $\varepsilon, \lambda$, and $\theta$ are three positive constant parameters. The first term is divided by the Hubble parameter $H$ in order that all the three terms have the same dimensions. The energy density given in (1) can be considered as an extension and generalization of other two DE models widely studied in recent time, that is, the Ricci $\mathrm{DE}(\mathrm{RDE})$ model and the DE energy density with Granda-Oliveros cut-off. In fact, in the limiting case corresponding to $\varepsilon=0$, we obtain the energy density of DE with Granda-Oliveros cut-off, and in the limiting case corresponding to $\varepsilon=0, \lambda=1$, and $\theta=2$, we recover the $\mathrm{RDE}$ model for flat universe (i.e., with curvature parameter $k$ equal to zero).

In this work we are considering DE interacting with pressureless DM which has energy density $\rho_{m}$. Various forms of interacting DE models have been constructed in order to fulfil the observational requirements. Many different works are presently available where the interacting DE have been discussed in detail. Some examples of interacting DE are presented in [35-40].

This work aims to reconstruct the DE model considered under $f(R, T)$ gravity and it is organized as follows. In Section 2, we describe the main features of the $f(R, T)=$ $\mu R+\nu T$ model. In Section 3, we consider the energy density of DE given in (1) in the context of $f(R, T)$ gravity considering the particular model considered. In Section 4, we study the statefinder parameters $r$ and $s$ for the energy density model we are considering in this work. In Section 5, we write a detailed discussion about the results found in this work. Finally, in Section 6, we write the Conclusions of this work.

\section{The $f(R, T)=\mu R+\nu T$ Model}

The metric of a spatially flat, homogeneous, and isotropic universe in Friedmann-Lemaitre-Robertson-Walker (FLRW) model is given by

$$
d s^{2}=d t^{2}-a^{2}(t)\left[d r^{2}+r^{2}\left(d \theta^{2}+\sin ^{2} \theta d \varphi^{2}\right)\right],
$$

where $a(t)$ represents a dimensionless scale factor (which gives information about the expansion of the universe), $t$ indicates the cosmic time, $r$ represents the radial component, and $\theta$ and $\varphi$ are the two angular coordinates.

We also know that the tetrad orthonormal components $e_{i}\left(x^{\mu}\right)$ are related to the metric through the following relation:

$$
g_{\mu \nu}=\eta_{i j} e_{\mu}^{i} e_{\nu}^{j}
$$

The Einstein field equations are given by

$$
\begin{gathered}
H^{2}=\frac{1}{3} \rho, \\
\dot{H}=-\frac{1}{2}(\rho+p),
\end{gathered}
$$

where $\rho$ and $p$ indicate (choosing units of $8 \pi G=c=1$ ) the total energy density and the total pressure, respectively. The conservation equation is given by

$$
\dot{\rho}+3 H(\rho+p)=0
$$

where

$$
\begin{gathered}
\rho=\rho_{\mathrm{DE}}+\rho_{m}, \\
p=p_{\mathrm{DE}} .
\end{gathered}
$$

We must emphasize here that we are considering pressureless DM $\left(p_{m}=0\right)$. Since the components do not satisfy the conservation equation separately in presence of interaction, we reconstruct the conservation equation by introducing an interaction term $Q$ which can be expressed in any of the following forms [41]: $Q \propto H \rho_{\mathrm{DE}}, Q \propto H \rho_{m}$, and $Q \propto H\left(\rho_{m}+\rho_{\mathrm{DE}}\right)$.

In this paper, we consider as interaction term the second of the three forms mentioned above. Accordingly, the conservation equation is reconstructed as

$$
\begin{gathered}
\dot{\rho}_{\mathrm{DE}}+3 H\left(\rho_{\mathrm{DE}}+p_{\mathrm{DE}}\right)=3 H \delta \rho_{m}, \\
\dot{\rho}_{m}+3 H \rho_{m}=-3 H \delta \rho_{m},
\end{gathered}
$$

where $\delta$ indicates an interaction constant parameter which gives information about the strength of the interaction between DE and DM. The present day value of $\delta$ is still not known exactly and it is under debate. 
One of the most interesting models of $f(R, T)$ gravity is the so-called $M_{37}$-model, whose action $S$ is given by [29]

$$
S=\int f(R, T) e d^{4} x+\int L_{m} e d^{4} x
$$

where $e$ is defined as $e=\operatorname{det}\left(e_{\mu}^{i}\right)=\sqrt{-g}$ (with $g$ being the determinant of the metric tensor $\left.g_{\mu \nu}\right), L_{m}$ is the matter Lagrangian, $R$ is the curvature scalar, and $T$ is the torsion scalar.

In this paper, we consider the following expressions for the curvature scalar $R$ and for the torsion scalar $T$ given, respectively, by

$$
\begin{gathered}
R=u+6\left(\dot{H}+2 H^{2}\right), \\
T=v-6 H^{2} .
\end{gathered}
$$

We now consider the particular case corresponding to $u=u(a, \dot{a})$ and $v=v(a, \dot{a})$, where $\dot{a}$ is the derivative of the scale factor with respect to the cosmic time $t$. Moreover, the scale factor $a(t)$, the torsion scalar $T$, and the curvature scalar $R$ are considered as independent dynamical variables. Then, after some algebraic calculations, the action given in (9) can be rewritten as

$$
S_{37}=\int d t L_{37}
$$

where the Lagrangian $L_{37}$ is given by

$$
\begin{gathered}
L_{37}=a^{3}\left(f-T f_{T}-R f_{R}+v f_{T}+u f_{R}\right), \\
-6\left(f_{R}+f_{T}\right) a \dot{a}^{2}-6\left(f_{R R} \dot{R}+f_{R T} \dot{T}\right) a^{2} \dot{a}-a^{3} L_{m} .
\end{gathered}
$$

The quantities $f_{R}, f_{T}, f_{R R}$, and $f_{R T}$ are, respectively, the first derivative of $f$ with respect to $R$, the first derivative of $f$ with respect to $T$, the second derivative of $f$ with respect to $R$, and the second derivative of $f$ with respect to $R$ and $T$.

The equations of $f(R, T)$ gravity are usually more complicated with respect to the equations of Einstein's theory of general relativity even if the FLRW metric is considered. For this reason, as stated before, we consider the following simple particular model of $f(R, T)$ gravity:

$$
f(R, T)=\mu R+\nu T,
$$

with $\mu$ and $\nu$ being two constant parameters.

The equations system of this model of $f(R, T)$ gravity is given by

$$
\begin{gathered}
\mu D_{1}+v E_{1}+K(\mu R+\nu T)=-2 a^{3} \rho, \\
\mu A_{1}+\nu B_{1}+M(\mu R+\nu T)=6 a^{2} p, \\
\dot{\rho}+3 H(\rho+p)=0,
\end{gathered}
$$

where

$$
\begin{gathered}
D_{1}=-6 a \dot{a}^{2}+a^{3} u_{\dot{a}} \dot{a}-a^{3}(u-R) \\
=6 a^{2} \ddot{a}+a^{3} \dot{a} u_{\dot{a}}=a^{3}\left(6 \frac{\ddot{a}}{a}+\dot{a} u_{\dot{a}}\right), \\
E_{1}=-6 a \dot{a}^{2}+a^{3} \dot{a} v_{\dot{a}}-a^{3}(v-T) \\
=-12 a \dot{a}^{2}+a^{3} \dot{a} v_{\dot{a}}=a^{3}\left(-12 \frac{\dot{a}^{2}}{a^{2}}+\dot{a} v_{\dot{a}}\right), \\
K=-a^{3}, \\
A_{1}=12 \dot{a}^{2}+6 a \ddot{a}+3 a^{2} \dot{a} u_{\dot{a}}+a^{3} u_{\dot{a}}-a^{3} u_{a}, \\
B_{1}=-24 \dot{a}^{2}-12 a \ddot{a}+3 a^{2} \dot{a} v_{\dot{a}}+a^{3} v_{\dot{a}}-a^{3} v_{a}, \\
M=-3 a^{2} .
\end{gathered}
$$

We get from (14)

$$
\begin{gathered}
-6(\mu+v) \frac{\dot{a}^{2}}{a^{2}}+\mu \dot{a} u_{\dot{a}}+v \dot{a} v_{\dot{a}}-\mu u-v v=-2 \rho, \\
-2(\mu+v)\left(\frac{\dot{a}^{2}}{a^{2}}+2 \frac{\ddot{a}}{a}\right)+\mu \dot{a} u_{\dot{a}}+v \dot{a} v_{\dot{a}}-\mu u \\
-v v+\frac{\mu}{3} a\left(\dot{u}_{\dot{a}}-u_{a}\right)+\frac{\nu}{3} a\left(\dot{v}_{\dot{a}}-v_{a}\right)=2 p, \\
\dot{\rho}+3 H(\rho+p)=0 .
\end{gathered}
$$

Then (16) can be rewritten as follows:

$$
\begin{gathered}
3(\mu+\nu) \frac{\dot{a}^{2}}{a^{2}}-\frac{1}{2}\left(\mu \dot{a} u_{\dot{a}}+v \dot{a} v_{\dot{a}}-\mu u-\nu v\right)=\rho, \\
(\mu+\nu)\left(\frac{\dot{a}^{2}}{a^{2}}+2 \frac{\ddot{a}}{a}\right)-\frac{1}{2}\left(\mu \dot{a} u_{\dot{a}}+\nu \dot{a} v_{\dot{a}}-\mu u-\nu v\right) \\
-\frac{\mu}{6} a\left(\dot{u}_{\dot{a}}-u_{a}\right)-\frac{v}{6} a\left(\dot{v}_{\dot{a}}-v_{a}\right)=-p, \\
\dot{\rho}+3 H(\rho+p)=0,
\end{gathered}
$$

or equivalently

$$
\begin{gathered}
3(\mu+\nu) H^{2}-\frac{1}{2}\left(\mu \dot{a} u_{\dot{a}}+v \dot{a} v_{\dot{a}}-\mu u-\nu v\right)=\rho \\
(\mu+\nu)\left(2 \dot{H}+3 H^{2}\right)-\frac{1}{2}\left(\mu \dot{a} u_{\dot{a}}+v \dot{a} v_{\dot{a}}-\mu u-v v\right) \\
-\frac{\mu}{6} a\left(\dot{u}_{\dot{a}}-u_{a}\right)-\frac{v}{6} a\left(\dot{v}_{\dot{a}}-v_{a}\right)=-p \\
\dot{\rho}+3 H(\rho+p)=0 .
\end{gathered}
$$

The above system has two equations and five unknown functions, which are $a, \rho, p, u$, and $v$.

We now assume the following expressions for $u$ and, $v$ :

$$
\begin{aligned}
& u=\alpha a^{n}, \\
& v=\beta a^{m},
\end{aligned}
$$


where $m, n, \alpha$, and $\beta$ are real constants. We also have that $u$ and $v$ can be expressed as

$$
\begin{aligned}
& u=\alpha\left(\frac{v}{\beta}\right)^{n / m}, \\
& v=\beta\left(\frac{u}{\alpha}\right)^{m / n} .
\end{aligned}
$$

Then, the system made by (18) leads to

$$
\begin{gathered}
3(\mu+\nu) H^{2}+\frac{1}{2}\left(\mu \alpha a^{n}+\nu \beta a^{m}\right)=\rho \\
(\mu+\nu)\left(2 \dot{H}+3 H^{2}\right)+\frac{\mu \alpha(n+3)}{6} a^{n}+\frac{\nu \beta(m+3)}{6} a^{m}=-p \\
\dot{\rho}+3 H(\rho+p)=0 .
\end{gathered}
$$

Finally, we have that the EoS parameter $\omega$ for this model is given by the relation

$$
\omega=\frac{p}{\rho}=-1-\frac{2(\mu+\nu) \dot{H}-(\mu / 6) a\left(\dot{u}_{\dot{a}}-u_{a}\right)-(\nu / 6) a\left(\dot{v}_{\dot{a}}-v_{a}\right)}{3(\mu+\nu) H^{2}-(1 / 2)\left(\mu \dot{a} u_{\dot{a}}+v \dot{a} v_{\dot{a}}-\mu u-v v\right)} .
$$

\section{Interacting DE in $f(R, T)$ Gravity}

Solving the differential equation for $\rho_{m}$ given in (8), we derive the following expression for $\rho_{m}$ :

$$
\rho_{m}=\rho_{m 0} a^{-3(1+\delta)},
$$

where $\rho_{m 0}$ indicates the present day value of $\rho_{m}$

Using (1) and (25) in the right-hand side of (21), we obtain the following expression of $H^{2}$ as function of the scale factor:

$$
\begin{aligned}
H^{2}= & C_{1} a^{-\left(\lambda+\sqrt{\lambda^{2}-8 \varepsilon[\theta-3(\mu+\nu)]}\right) / 2 \varepsilon} \\
& +C_{2} a^{\left(-\lambda+\sqrt{\lambda^{2}-8 \varepsilon[\theta-3(\mu+\nu)]}\right) / 2 \varepsilon} \\
& +\frac{\alpha \mu a^{n}}{n^{2} \varepsilon+n \lambda+2[\theta-3(\mu+\nu)]} \\
& +\frac{\beta \nu a^{m}}{m^{2} \varepsilon+m \lambda+2[\theta-3(\mu+\nu)]} \\
& -\left(2 a^{-3(1+\delta)} \rho_{m 0}\right) \\
& \times\left(9(1+\delta)^{2} \varepsilon+2 \theta\right. \\
& \quad-3[\lambda(1+\delta)+2(\mu+\nu)])^{-1}
\end{aligned}
$$

where $C_{1}$ and $C_{2}$ are two constants of integration.

In order to have a real and definite expression of $\mathrm{H}^{2}$ given in (26), the following conditions must be satisfied:

(1) $\varepsilon \neq 0$,

(2) $\lambda^{2}-8 \varepsilon[\theta-3(\mu+\nu)] \geq 0$,
(3) $n^{2} \varepsilon+n \lambda+2[\theta-3(\mu+\nu)] \neq 0$,

(4) $m^{2} \varepsilon+m \lambda+2[\theta-3(\mu+\nu)] \neq 0$,

(5) $9(1+\delta)^{2}+2 \theta-3[\lambda(1+\delta)+2(\mu+\nu)] \neq 0$.

We can now derive the expressions of the first and the second time derivative of the Hubble parameter $H$, that is, $\dot{H}$ and $\ddot{H}$, as functions of the scale factor $a$ differentiating (26) with respect to the cosmic time $t$ :

$$
\begin{aligned}
\dot{H}= & -\frac{C_{1}}{2}\left(\frac{\lambda+\sqrt{\lambda^{2}-8 \varepsilon[\theta-3(\mu+\nu)]}}{2 \varepsilon}\right) \\
& \times a^{-\left(\lambda+\sqrt{\lambda^{2}-8 \varepsilon[\theta-3(\mu+\nu)]} / 2 \varepsilon\right.} \\
& +\frac{C_{2}}{2}\left(\frac{-\lambda+\sqrt{\lambda^{2}-8 \varepsilon[\theta-3(\mu+\nu)]}}{2 \varepsilon}\right) \\
& \times a^{\left(-\lambda+\sqrt{\lambda^{2}-8 \varepsilon[\theta-3(\mu+\nu)]} / 2 \varepsilon\right.} \\
& +\frac{n \alpha \mu a^{n}}{2\left\{n^{2} \varepsilon+n \lambda+2[\theta-3(\mu+\nu)]\right\}} \\
& +\frac{m \beta \nu a^{m}}{2\left\{m^{2} \varepsilon+m \lambda+2[\theta-3(\mu+\nu)]\right\}} \\
& +\left(3(1+\delta) a^{-3(1+\delta)} \rho_{m 0}\right) \\
& \times\left(9(1+\delta)^{2} \varepsilon+2 \theta\right. \\
& -3[\lambda(1+\delta)+2(\mu+\nu)])^{-1}
\end{aligned}
$$

$$
\ddot{H}=H\left\{\frac{C_{1}}{2}\left(\frac{\lambda+\sqrt{\lambda^{2}-8 \varepsilon[\theta-3(\mu+\nu)]}}{2 \varepsilon}\right)^{2}\right.
$$

$$
\begin{aligned}
& \times a^{-\left(\lambda+\sqrt{\lambda^{2}-8 \varepsilon[\theta-3(\mu+\nu)]}\right) / 2 \varepsilon} \\
& +\frac{C_{2}}{2}\left(\frac{-\lambda+\sqrt{\lambda^{2}-8 \varepsilon[\theta-3(\mu+\nu)]}}{2 \varepsilon}\right)^{2} \\
& \times a^{\left(-\lambda+\sqrt{\lambda^{2}-8 \varepsilon[\theta-3(\mu+\nu)]} / 2 \varepsilon\right.} \\
& +\frac{n^{2} \alpha \mu a^{n}}{2\left\{n^{2} \varepsilon+n \lambda+2[\theta-3(\mu+\nu)]\right\}} \\
& +\frac{m^{2} \beta \nu a^{m}}{2\left\{m^{2} \varepsilon+m \lambda+2[\theta-3(\mu+\nu)]\right\}} \\
& -\left(9(1+\delta)^{2} a^{-3(1+\delta)} \rho_{m 0}\right) \\
& \times\left(9(1+\delta)^{2} \varepsilon+2 \theta\right. \\
& \left.\quad-3[\lambda(1+\delta)+2(\mu+\nu)])^{-1}\right\}
\end{aligned}
$$


Using (26), (27), and (28) in (1), we obtain the following expression of the energy density $\rho_{\mathrm{DE}}$ :

$$
\begin{aligned}
\rho_{\mathrm{DE}}=\frac{1}{2}[ & \frac{\left(n^{2} \varepsilon+2 \theta+n \lambda\right) \alpha \mu a^{n}}{\left\{n^{2} \varepsilon+n \lambda+2[\theta-3(\mu+\nu)]\right\}} \\
& +\frac{\left(m^{2} \varepsilon+2 \theta+2 m \lambda\right) \beta \nu a^{m}}{\left\{m^{2} \varepsilon+m \lambda+2[\theta-3(\mu+\nu)]\right\}} \\
& +6 C_{1}(\mu+\nu) a^{-\left(\lambda+\sqrt{\lambda^{2}-8 \varepsilon[\theta-3(\mu+\nu)]}\right) / 2 \varepsilon} \\
& +6 C_{2}(\mu+\nu) a^{\left(-\lambda+\sqrt{\lambda^{2}-8 \varepsilon[\theta-3(\mu+\nu)]}\right) / 2 \varepsilon} \\
& -\left(2\left[9(1+\delta)^{2} \varepsilon+2 \theta-3(1+\delta) \lambda\right] a^{-3(1+\delta)} \rho_{m 0}\right) \\
& \times\left(9(1+\delta)^{2} \varepsilon+2 \theta\right. \\
& \left.-3[\lambda(1+\delta)+2(\mu+\nu)])^{-1}\right] .
\end{aligned}
$$

Taking into account the expression of $\rho_{\mathrm{DE}}$ given in (29), we derive that the expression of the pressure $p_{\mathrm{DE}}$ of $\mathrm{DE}$ is given by

$$
\begin{aligned}
p_{\mathrm{DE}}= & C_{1}(\mu+\nu)\left(\frac{-6 \varepsilon+\lambda+\sqrt{\lambda^{2}-8 \varepsilon[\theta-3(\mu+\nu)]}}{2 \varepsilon}\right) \\
& \times a^{-\left(\lambda+\sqrt{\lambda^{2}-8 \varepsilon[\theta-3(\mu+\nu)]}\right) / 2 \varepsilon} \\
& \times C_{2}(\mu+\nu)\left(\frac{6 \varepsilon-\lambda+\sqrt{\lambda^{2}-8 \varepsilon[\theta-3(\mu+\nu)]}}{2 \varepsilon}\right) \\
& \times a^{\left(-\lambda+\sqrt{\lambda^{2}-8 \varepsilon[\theta-3(\mu+\nu)]} / 2 \varepsilon\right.} \\
& -\left([2 \theta+n(n \varepsilon+\lambda)](n+3) \alpha \mu a^{n}\right) \\
& \times\left(6\left\{n^{2} \varepsilon+n \lambda+2[\theta-3(\mu+\nu)]\right\}\right)^{-1} \\
& -\left([2 \theta+n(n \varepsilon+\lambda)](m+3) \beta \nu a^{m}\right) \\
& \times\left(6\left\{m^{2} \varepsilon+m \lambda+2[\theta-3(\mu+\nu)]\right\}\right)^{-1} \\
& -\left(\left[9(1+\delta)^{2} \varepsilon+2 \theta-3(1+\delta) \lambda\right] a^{-3(1+\delta)} \delta \rho_{m 0}\right) \\
& \times\left(9(1+\delta)^{2} \varepsilon+2 \theta-3[\lambda(1+\delta)+2(\mu+\nu)]\right)^{-1} .
\end{aligned}
$$

Using the expressions of the energy density $\rho_{\mathrm{DE}}$ and the pressure $p_{\mathrm{DE}}$ of DE given, respectively, in (29) and (30), and the expression of $\rho_{m}$ given in (25), we get the EoS parameter $\omega_{\mathrm{DE}}$ for $\mathrm{DE}$ and the total EoS parameter $\omega_{\text {tot }}$ as follows:

$$
\begin{gathered}
\omega_{\mathrm{DE}}=\frac{p_{\mathrm{DE}}}{\rho_{\mathrm{DE}}}, \\
\omega_{\mathrm{tot}}=\frac{p_{\mathrm{DE}}}{\rho_{\mathrm{DE}}+\rho_{m}} .
\end{gathered}
$$

We must remember here that we are considering the case of pressureless DM, so that $p_{m}=0$.

We now want to consider the properties of the deceleration parameter $q$ for the model we are considering. The deceleration parameter $q$ is generally defined as follows:

$$
q=-1-\frac{a \ddot{a}}{\dot{a}^{2}}=-1-\frac{\dot{H}}{H^{2}},
$$

where the expressions of $H^{2}$ and $\dot{H}$ are given, respectively, in (26) and (27). The deceleration parameter, the Hubble parameter $H$, and the dimensionless energy density parameters $\Omega_{\mathrm{DE}}, \Omega_{m}$, and $\Omega_{k}$ (which will be considered and studied in the following Sections) are a set of useful parameters if it is needed to describe cosmological observations.

\section{The Statefinder Parameters}

In order to have a better comprehension of the properties of the DE model taken into account, we can compare it with a model independent diagnostics which is able to differentiate between a wide variety of dynamical DE models, including the $\Lambda \mathrm{CDM}$ model. We consider here the diagnostic, also known as statefinder diagnostic, which introduces a pair of parameters $\{r, s\}$ defined, respectively, as follows:

$$
\begin{gathered}
r=1+3 \frac{\dot{H}}{H^{2}}+\frac{\ddot{H}}{H^{3}}=1+\frac{3 \dot{H}+\ddot{H} / H}{H^{2}}, \\
s=-\frac{3 H \dot{H}+\ddot{H}}{3 H\left(2 \dot{H}+3 H^{2}\right)}=-\frac{3 \dot{H}+\ddot{H} / H}{3\left(2 \dot{H}+3 H^{2}\right)} .
\end{gathered}
$$

Using (26), (27), and (29), we get the statefinder parameters as

$$
\begin{gathered}
r=1+\frac{\rho_{1}}{\rho_{2}}, \\
s=\frac{\zeta_{1}}{\zeta_{2}},
\end{gathered}
$$

with

$$
\begin{aligned}
\rho_{1}= & C_{1}(\mu+\nu)\left(\lambda+\sqrt{\lambda^{2}-8 \varepsilon[\theta-3(\mu+\nu)]}\right) \\
& \times\left(\frac{-6 \varepsilon+\lambda+\sqrt{\lambda^{2}-8 \varepsilon[\theta-3(\mu+\nu)]}}{8 \varepsilon^{2}}\right) \\
& \times a^{-\left(\lambda+\sqrt{\lambda^{2}-8 \varepsilon[\theta-3(\mu+\nu)]} / 2 \varepsilon\right.} \\
& +C_{2}(\mu+\nu)\left(-\lambda+\sqrt{\lambda^{2}-8 \varepsilon[\theta-3(\mu+\nu)]}\right)
\end{aligned}
$$




$$
\begin{aligned}
& \times\left(\frac{6 \varepsilon-\lambda+\sqrt{\lambda^{2}-8 \varepsilon[\theta-3(\mu+\nu)]}}{8 \varepsilon^{2}}\right) \\
& \times a^{\left(-\lambda+\sqrt{\lambda^{2}-8 \varepsilon[\theta-3(\mu+\nu)]}\right) / 2 \varepsilon} \\
& +\frac{(n+3) n \alpha \mu a^{n}}{2\left\{n^{2} \varepsilon+n \lambda+2[\theta-3(\mu+\nu)]\right\}} \\
& +\frac{(m+3) m \beta v a^{m}}{2\left\{m^{2} \varepsilon+m \lambda+2[\theta-3(\mu+\nu)]\right\}} \\
& -\left(9 a^{-3(1+\delta)} \delta(1+\delta) \rho_{m 0}\right) \\
& \times\left(9(1+\delta)^{2} \varepsilon+2 \theta\right. \\
& -3[\lambda(1+\delta)+2(\mu+\nu)])^{-1}, \\
& \rho_{2}=C_{1} a^{-\left(\lambda+\sqrt{\lambda^{2}-8 \varepsilon[\theta-3(\mu+\nu)]}\right) / 2 \varepsilon} \\
& +C_{2} a^{\left(-\lambda+\sqrt{\lambda^{2}-8 \varepsilon[\theta-3(\mu+\nu)]}\right) / 2 \varepsilon} \\
& +\frac{\alpha \mu a^{n}}{n^{2} \varepsilon+n \lambda+2[\theta-3(\mu+\nu)]} \\
& +\frac{\beta v a^{m}}{m^{2} \varepsilon+m \lambda+2[\theta-3(\mu+\nu)]} \\
& -\left(2 a^{-3(1+\delta)} \rho_{m 0}\right) \\
& \times\left(9(1+\delta)^{2} \varepsilon+2 \theta\right. \\
& -3[\lambda(1+\delta)+2(\mu+\nu)])^{-1}, \\
& \zeta_{1}=-C_{1}(\mu+\nu)\left(\lambda+\sqrt{\lambda^{2}-8 \varepsilon[\theta-3(\mu+\nu)]}\right) \\
& \times\left(\frac{-6 \varepsilon+\lambda+\sqrt{\lambda^{2}-8 \varepsilon[\theta-3(\mu+\nu)]}}{8 \varepsilon^{2}}\right) \\
& \times a^{-\left(\lambda+\sqrt{\lambda^{2}-8 \varepsilon[\theta-3(\mu+\nu)]}\right) / 2 \varepsilon} \\
& -C_{2}(\mu+\nu)\left(-\lambda+\sqrt{\lambda^{2}-8 \varepsilon[\theta-3(\mu+\nu)]}\right) \\
& \times\left(\frac{6 \varepsilon-\lambda+\sqrt{\lambda^{2}-8 \varepsilon[\theta-3(\mu+\nu)]}}{8 \varepsilon^{2}}\right) \\
& \times a^{\left(-\lambda+\sqrt{\lambda^{2}-8 \varepsilon[\theta-3(\mu+\nu)]}\right) / 2 \varepsilon} \\
& -\frac{(n+3) n \alpha \mu a^{n}}{2\left\{n^{2} \varepsilon+n \lambda+2[\theta-3(\mu+\nu)]\right\}} \\
& -\frac{(m+3) m \beta \nu a^{m}}{2\left\{m^{2} \varepsilon+m \lambda+2[\theta-3(\mu+\nu)]\right\}}
\end{aligned}
$$

$$
\begin{aligned}
+ & \left(9 a^{-3(1+\delta)} \delta(1+\delta) \rho_{m 0}\right) \\
\times & \left(9(1+\delta)^{2} \varepsilon+2 \theta\right. \\
& -3[\lambda(1+\delta)+2(\mu+\nu)])^{-1} \\
\zeta_{2}= & -3 C_{1}\left(\frac{-6 \varepsilon+\lambda+\sqrt{\lambda^{2}-8 \varepsilon[\theta-3(\mu+\nu)]}}{2 \varepsilon}\right) \\
& \times a^{-\left(\lambda+\sqrt{\lambda^{2}-8 \varepsilon[\theta-3(\mu+\nu)]}\right) / 2 \varepsilon} \\
+ & 3 C_{2}\left(\frac{6 \varepsilon-\lambda+\sqrt{\lambda^{2}-8 \varepsilon[\theta-3(\mu+\nu)]}}{2 \varepsilon}\right) \\
& \times a^{\left(-\lambda+\sqrt{\lambda^{2}-8 \varepsilon[\theta-3(\mu+\nu)]}\right) / 2 \varepsilon} \\
+ & 3 \frac{(n+3) \alpha \mu a^{n}}{\left\{n^{2} \varepsilon+n \lambda+2[\theta-3(\mu+\nu)]\right\}} \\
+ & 3 \frac{(m+3) \beta \nu a^{m}}{\left\{m^{2} \varepsilon+m \lambda+2[\theta-3(\mu+\nu)]\right\}} \\
+ & \left(18 a^{-3(1+\delta)} \delta \rho_{m 0}\right) \\
\times & \left(9(1+\delta)^{2} \varepsilon+2 \theta\right. \\
& -3[\lambda(1+\delta)+2(\mu+\nu)])^{-1}
\end{aligned}
$$

\section{Discussion}

In this Section, we discuss the behavior of the physical quantities derived in the previous sections. We have considered the following values for the parameters involved: $C_{1}=0.2$, $C_{2}=1.2, m=1.2, n=1.4, \beta=1.2, \alpha=1.5, \theta=0.002, \lambda=2$, $\nu=0.5, \mu=0.9, \delta=0.05$, and $\rho_{m 0}=0.23$. We considered three different cases corresponding to three different values of the parameter $\varepsilon$, that is, $\varepsilon=2,3$, and 4 .

In Figure 1, we plotted the expression of the Hubble parameter $H$, obtained from (26), as function of the redshift $z$. It is evident that the Hubble parameter $H$ has a decaying behavior with varying values of the parameter $\varepsilon$ and the redshift $z$ going from higher to lower redshifts. However, this decaying pattern is apparent till $z \approx-0.5$. In fact, in a very late stage $z>-0.5$, it shows an increasing pattern.

In Figure 2, we have plotted the time derivative of Hubble parameter $\dot{H}$ against the redshift $z$. We have observed that for $\varepsilon=3, \dot{H}$ transits from negative to positive side at $z \approx-0.5$. However, for $\varepsilon=2$ and 4 this transition occurs at lower redshift $z \approx-0.1$. In Figures 3 and 4 we have plotted, respectively, the equation of state (EoS) parameter for $\mathrm{DE}$, defined as $\omega_{\mathrm{DE}}=p_{\mathrm{DE}} / \rho_{\mathrm{DE}}$, and the effective EoS parameter, defined as $\omega_{\mathrm{eff}}=p_{\mathrm{DE}} /\left(\rho_{\mathrm{DE}}+\rho_{\mathrm{DM}}\right)$, for the three different values of $\varepsilon$ considered in this work. In Figure 3, 


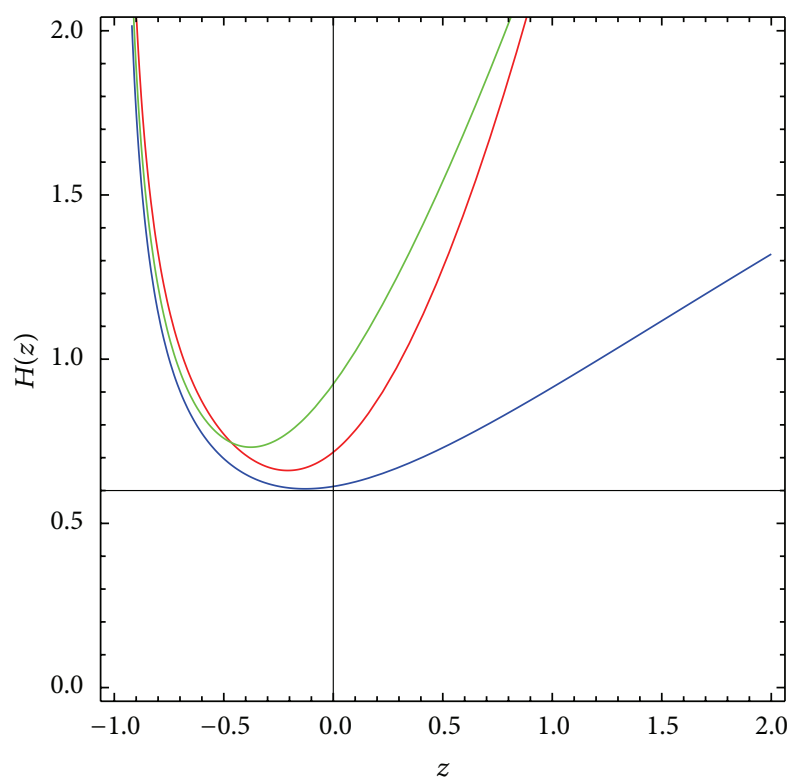

Figure 1: The Hubble parameter $H$, obtained from (26), as a function of redshift $z$. The red, green, and blue lines correspond to $\varepsilon=$ 2,3 , and 4 , respectively.

we have observed that for $\varepsilon=2, \omega_{\mathrm{DE}}$ crosses the phantom divide -1 at $z \approx 0$. For $\varepsilon=3$ the phantom divide is crossed at $z \approx-0.2$. However, for $\varepsilon=4$, the equation of state (EoS) parameter for DE stays below -1 . Thus, for $\varepsilon=2$ and 3 , $\omega_{\mathrm{DE}}$ transits from quintessence to phantom, that is, has a quintom-like behavior. Instead, for $\varepsilon=4$, the EoS parameter has a phantom-like behavior. In Figure 4, we have plotted the effective EoS parameter $\omega_{\text {eff. }}$. In this case, for all values of $\varepsilon$ considered, there is a crossing of phantom divide. Moreover, for $\varepsilon=4, \omega_{\text {eff }}$ crosses the phantom divide earlier with respect to the other cases, in particular for $z \approx 0.2$.

The deceleration parameter $q$ has been plotted as a function of $z$ in Figure 5. For $\varepsilon=2$, and 3, there is a transition from positive to negative $q$, that is, transition from decelerated to accelerated expansion. For $\varepsilon=3$, the deceleration parameter changes sign at $z=0$, and for $\varepsilon=2$, it changes $\operatorname{sign}$ at $z \approx 0.1$. However, for $\varepsilon=4$, the deceleration parameter always stays at negative level. Thus, for $\varepsilon=4$, we are getting ever-accelerating universe.

Next, we have plotted in Figure 6 the fractional density of $\mathrm{DE}$, given by $\Omega_{\mathrm{DE}}=\rho_{\mathrm{DE}} / 3 \widetilde{H}^{2}(z)$, and the fractional density of matter, given by $\Omega_{m}=\rho_{m} / 3 \widetilde{H}^{2}(z)$, against the redshift $z$. $\widetilde{H}^{2}$ is defined as $\widetilde{H}^{2}(z)=(\mu+\nu) H^{2}+(1 / 6)\left[\alpha \mu(1+z)^{-n}+\right.$ $\left.\beta \nu(1+z)^{-m}\right]$. The solid lines correspond to $\Omega_{\mathrm{DE}}$ and the dashed lines correspond to $\Omega_{\mathrm{DM}}$. In this Figure, there is a clear indication of transition of the universe from dark matter dominated phase to the dark energy dominated phase. At very early stage of the universe $z>1$, the dark energy density is largely dominated by dark matter density. We denote the cross-over point by $z_{\text {cross }}$ and it comes out to be $z_{\text {cross }} \approx 0.5$, that is, where $\Omega_{\mathrm{DE}}=\Omega_{\mathrm{DM}}$ for all values of $\varepsilon$ considered in this work. Hence, the $f(R, T)$ model, based on which we have reconstructed $\mathrm{DE}$ density, is capable of achieving

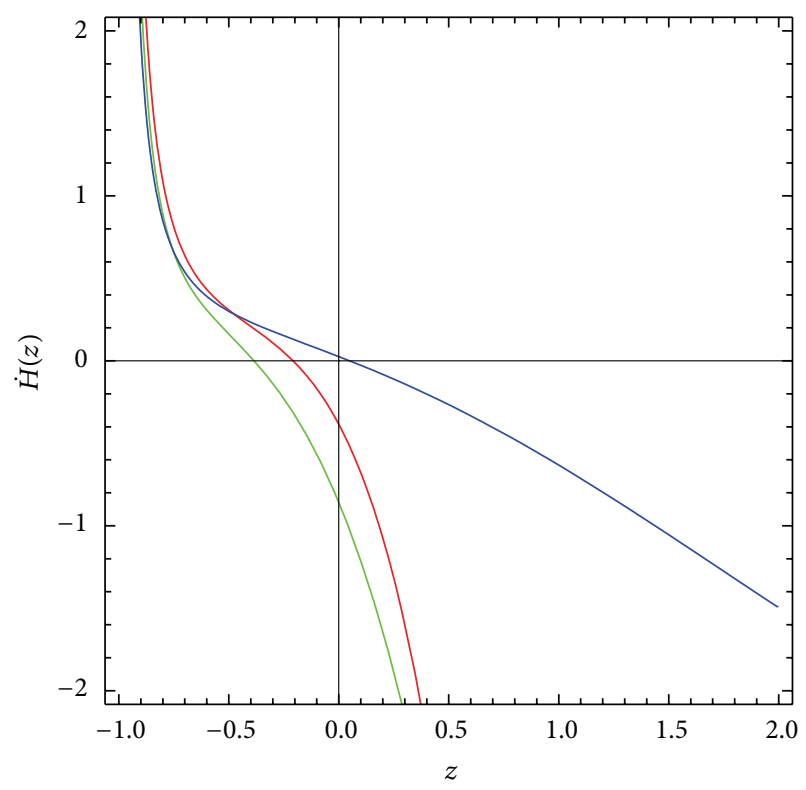

Figure 2: The time derivative of reconstructed Hubble parameter as a function of redshift $z$. The red, green and blue lines correspond to $\varepsilon=2,3$, and 4 , respectively.

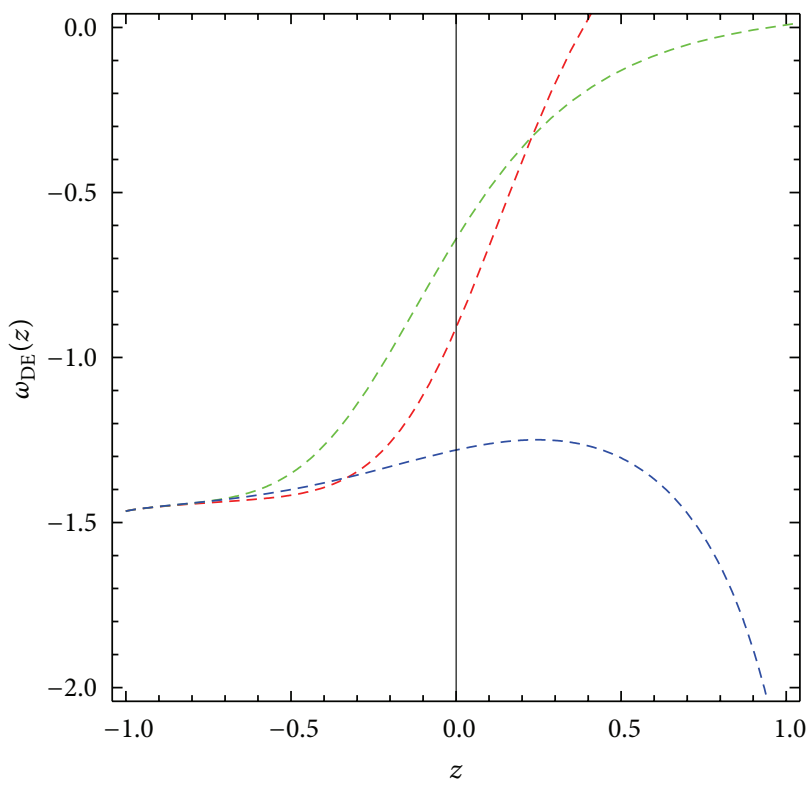

FIGURE 3: The EoS parameter $\omega_{\mathrm{DE}}$ for the reconstructed DE.

the present DE dominated universe from the earlier dark matter dominated universe.

Sahni et al. [42] recently demonstrated that the statefinder diagnostic is effectively able to discriminate between different models of DE. Chaplygin gas, braneworld, quintessence, and cosmological constant models were investigated by Alam et al. [43] using the statefinder diagnostics; they observed that the statefinder pair could differentiate between these different models. An investigation on statefinder parameters for differentiating between DE and modified 


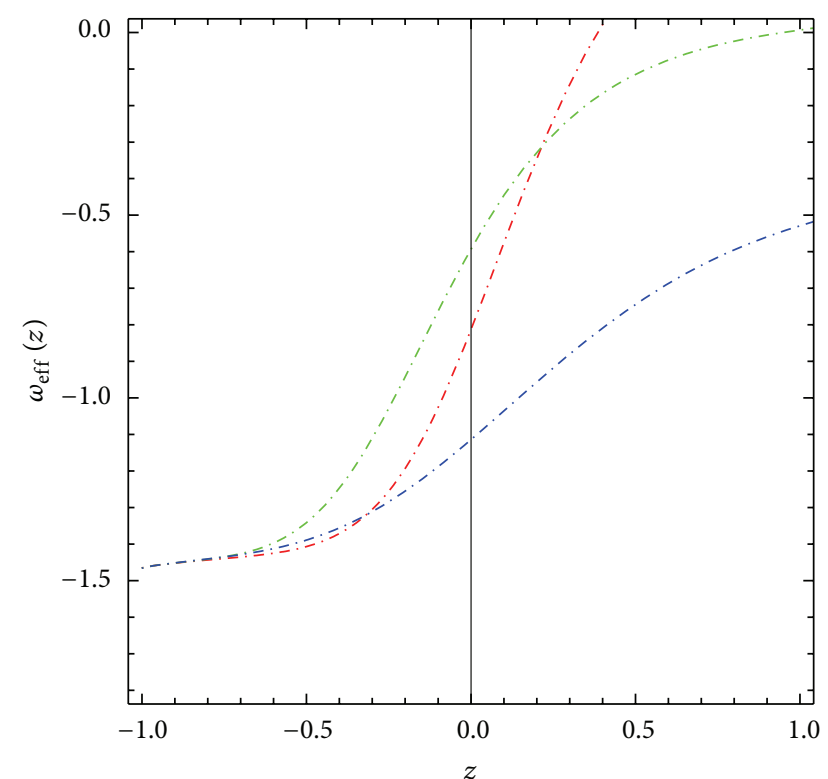

FIGURE 4: The effective EoS parameter $\omega_{\text {eff }}(z)=p_{\mathrm{DE}} /\left(\rho_{\mathrm{DE}}+\rho_{\mathrm{DM}}\right)$.

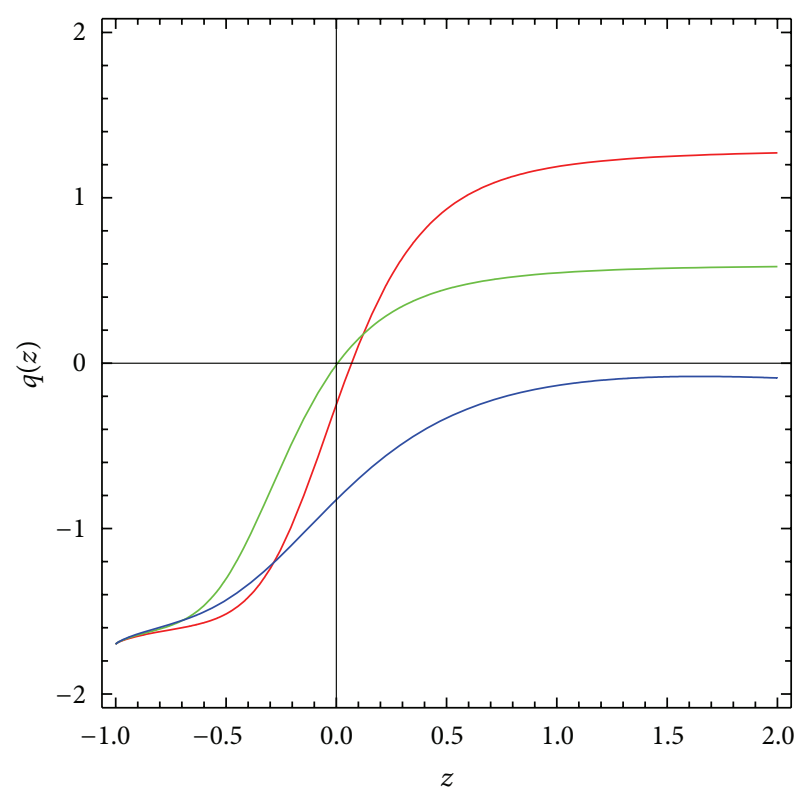

FIGURE 5: The deceleration parameter $q$ as a function of $z$.

gravity was carried out in [44]. Statefinder diagnostics for $f(T)$ gravity has been well studied in $\mathrm{Wu}$ and $\mathrm{Yu}$ [45]. In the $\{r, s\}$ plane, $s>0$ corresponds to a quintessence-like model of DE and $s<0$ corresponds to a phantom-like model of DE. Moreover, an evolution from phantom to quintessence or inverse is given by crossing of the fixed point $(r=1, s=0)$ in $\{r, s\}$ plane [45], which corresponds to $\Lambda$ CDM scenario. The statefinder parameters $\{r, s\}$ have been plotted in Figure 7 for different values of the parameter $\varepsilon$. It is clearly visible that the $\{r-s\}$ trajectories are converging towards the fixed point $\{r=$ $1, s=0\}\left.\right|_{\Lambda \mathrm{CDM}}$. Thus, the $f(R, T)$ model is capable of attaining the $\Lambda \mathrm{CDM}$ phase of the universe. Furthermore, for finite $r$,

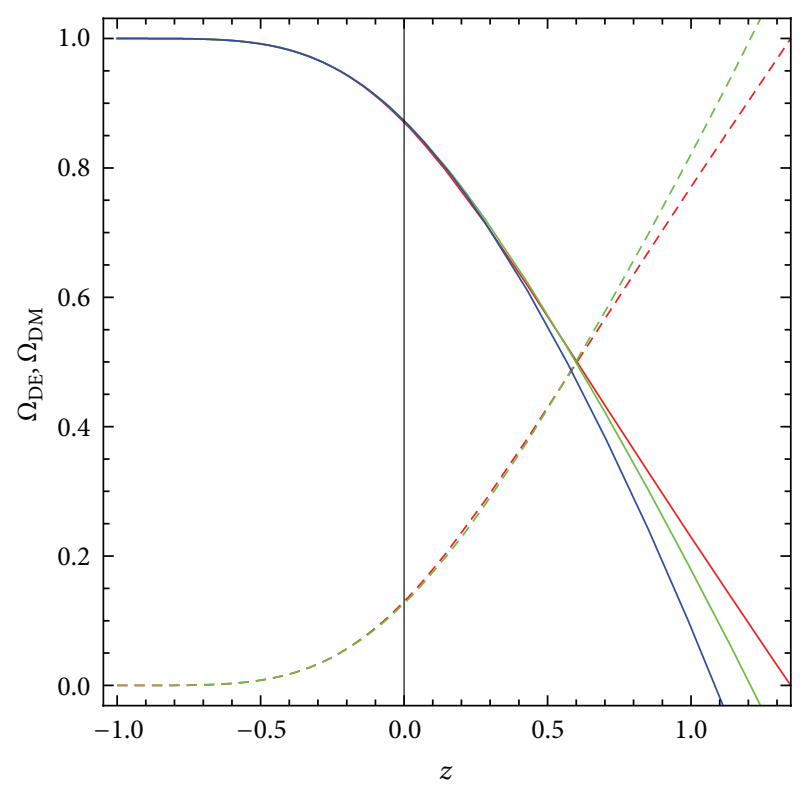

FIGURE 6: The fractional densities $\Omega_{\mathrm{DE}}$ (smooth lines) and $\Omega_{\mathrm{DM}}$ (dashed lines) as function of redshift $z$.

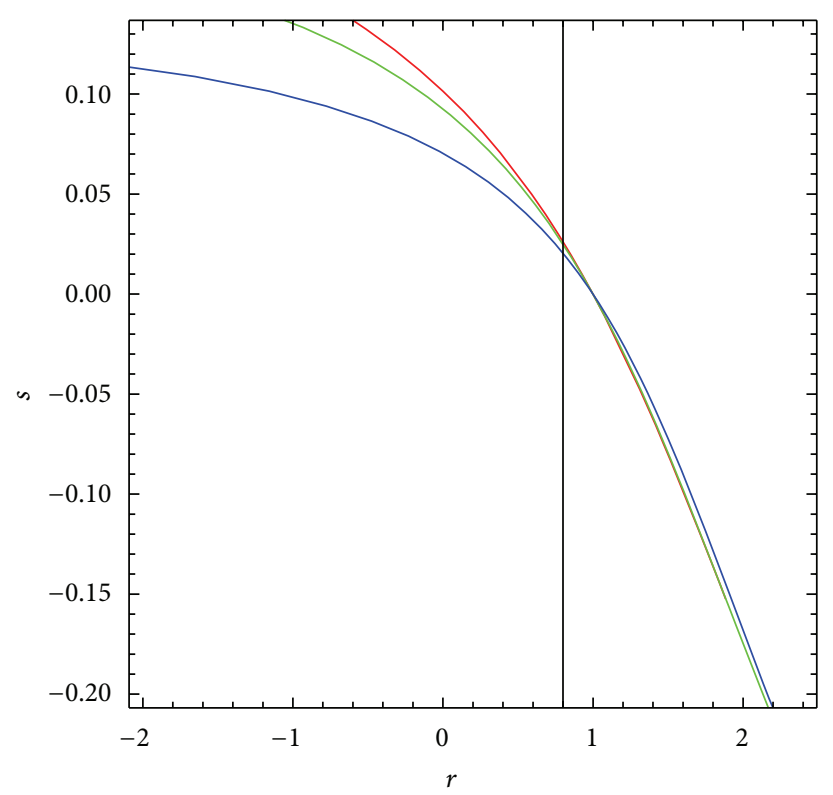

FIGURE 7: Statefinder trajectories for various choices of parameters.

$s \rightarrow-\infty$. Thus, the model can interpolate between dust and $\Lambda \mathrm{CDM}$ phase of the universe.

Finally, in Figure 8, we plotted the squared speed of the sound, defined as $v_{s}^{2}=\dot{p} / \dot{\rho}$, where the upper dot indicates derivative with respect to the cosmic time $t$ for the model we are considering as a function of $z$. The sign of the squared speed of sound is fundamental in order to study the stability of a background evolution. A negative value of $v_{s}^{2}$ implies a classical instability of a given perturbation in general relativity [46, 47]. Myung [47] recently observed that the squared speed of sound for HDE stays always negative if 


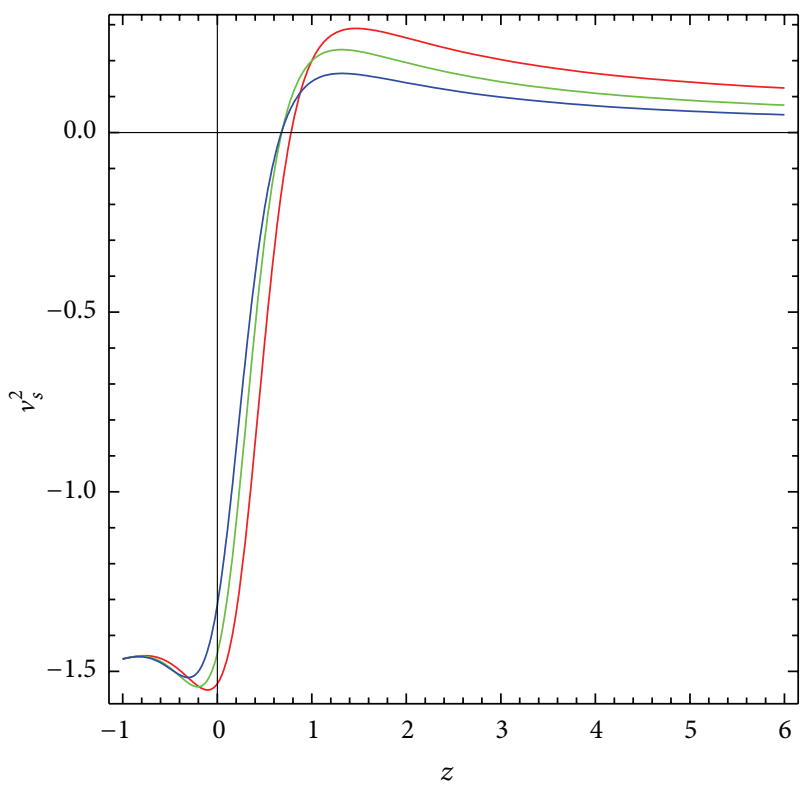

FIGURE 8: Squared speed of sound $v_{s}^{2}=\dot{p} / \dot{\rho}$ as a function of redshift $z$.

the future event horizon is considered as IR cutoff, while for Chaplygin gas and tachyon $v_{s}^{2}$ is observed to benonnegative. Kim et al. [46] found that $v_{s}^{2}$ for Agegraphic DE (ADE) stays always negative, which leads to the instability of the perfect fluid for the model. Moreover, it was found that the ghost QCD [48] DE model is unstable. In a recent work, Sharif and Jawad [49] have shown that interacting new HDE is characterized by negative squared speed of the sound.

In the recent work of Pasqua et al. [32], authors observed that the interacting modified holographic Ricci DE (MHRDE) model in $f(R, T)=\mu R+\nu T$ gravity is classically stable.

Jawad et al. [50] showed that $f(G)$ model in HDE scenario with power-law scale factor is classically unstable.

Pasqua et al. [51] showed that the DE model based on generalized uncertainty principle (GUP) with power-law form of the scale factor $a(t)$ is instable.

We have observed that, for all values of $\varepsilon, v_{s}^{2}$ is positive till the redshift $z \approx 0.5$. However, after this stage it enters the negative region. Thus, although the model is classically stable in the early universe, for present universe it is classically unstable.

\section{Concluding Remarks}

In this work, we have considered a recently proposed model of energy density of DE which depends on three terms, one proportional to the squared Hubble parameter $H$, one proportional to the time derivative of $H$, and one proportional to the second time derivative of $H$ interacting with pressureless DM in the framework of the $f(R, T)$ modified gravity theory for the special model given by $f(R, T)=$ $\mu R+\nu T$, where $\mu$ and $\nu$ represent two constants. The DE model considered here reduces to other two well-studied DE model (the Ricci DE model and the DE energy density model with Granda-Oliveros cut-off) for some particular values of the three parameters involved, that is, $\varepsilon, \lambda$, and $\theta$. We have derived the expressions and studied the behavior of some important physical quantities which gave useful hints about the model studied. The Hubble parameter $H$ exhibits a decaying behavior going from higher to lower redshifts until the redshift of about $z \approx-0.5$, when it starts to increase. The time derivative of the Hubble parameter, that is, $\dot{H}$, shows a transition from negative to positive values for different values of the redshift $z$ according to the value of $\varepsilon$ considered. We have observed that the equation of state (EoS) parameter of DE exhibits a transition from $\omega<-1$ to $\omega>-1$, that is, transition from quintessence to phantom (i.e., quintom) with the evolution of the universe for $\varepsilon=2$ and $\varepsilon=3$, instead it is always negative for $\varepsilon=4$. Moreover, the effective equation of state (EoS) parameter $\omega_{\text {eff }}$ always shows a transition from quintessence to phantom. Hence, we can conclude that the reconstructed DE model based on the $f(R, T)$ gravity model considered leads to an equation of state $(\mathrm{EoS})$ parameter that has a quintom-like behavior. We have further observed that the said model is capable of attaining the dark energy dominated accelerated phase of the universe from dark matter dominated decelerated phase of the universe. Through statefinder trajectories we have shown that the $f(R, T)$ is capable of attaining the $\Lambda$ CDM phase of the universe and can interpolate between dust and $\Lambda C D M$ phase of the universe. Through squared speed of sound we have seen that the model under consideration is classically stable in the earlier stage of the universe but classically unstable in the current stage.

\section{Conflict of Interests}

The authors declare that there is no conflict of interests regarding the publication of this paper.

\section{Acknowledgment}

The second author acknowledges financial support from the Department of Science and Technology, Govtvernment of India, under project Grant no. SR/FTP/PS-167/2011.

\section{References}

[1] D. N. Spergel, L. Verde, H. V. Peiris et al., "First-year Wilkinson Microwave Anisotropy Probe (WMAP) observations: determination of cosmological parameters," Astrophysical Journal, Supplement Series, vol. 148, no. 1, pp. 175-194, 2003.

[2] S. Perlmutter, G. Aldering, G. Goldhaber et al., "Measurements of $\Omega$ and $\Lambda$ from 42 high-redshift supernovae," Astrophysical Journal Letters, vol. 517, no. 2, pp. 565-586, 1999.

[3] D. Polarski, "Some views on dark energy," in Dark Energy: Observational and Theoretical Approaches, P. Ruiz-Lapuente, Ed., Cambridge University Press, Cambridge, UK, 2010.

[4] E. J. Copeland, M. Sami, and S. Tsujikawa, "Dynamics of dark energy," International Journal of Modern Physics D, vol. 15, no. 11, pp. 1753-1935, 2006. 
[5] T. Padmanabhan, "Dark energy: the cosmological challenge of the millennium," Current Science, vol. 88, no. 7, pp. 1057-1067, 2005.

[6] K. Bamba, S. Capozziello, S. Nojiri, and S. D. Odintsov, "Dark energy cosmology: the equivalent description via different theoretical models and cosmography tests," Astrophysics and Space Science, vol. 342, no. 1, pp. 155-228, 2012.

[7] S. Nojiri and S. D. Odintsov, "Introduction to modified gravity and gravitational alternative for dark energy," International Journal of Geometric Methods in Modern Physics, vol. 4, no. 1, pp. 115-145, 2007.

[8] T. Clifton, P. G. Ferreira, A. Padilla, and C. Skordis, "Modified gravity and cosmology," Physics Reports, vol. 513, no. 1-3, pp. 1189, 2012.

[9] S. Nojiri, S. D. Odintsov, and H. Štefančić, "Transition from a matter-dominated era to a dark energy universe," Physical Review D, vol. 74, no. 8, Article ID 086009, 2006.

[10] S. Nojiri and S. D. Odintsov, "Modified $f(R)$ gravity unifying $R^{m}$ inflation with the $\Lambda \mathrm{CDM}$ epoch," Physical Review D, vol. 77, Article ID 026007, 7 pages, 2008.

[11] Y.-F. Cai, S.-H. Chen, J. B. Dent, S. Dutta, and E. N. Saridakis, "Matter bounce cosmology with the $f(T)$ gravity," Classical and Quantum Gravity, vol. 28, no. 21, Article ID 215011, 2011.

[12] R. Ferraro and F. Fiorini, "Modified teleparallel gravity: inflation without an inflaton," Physical Review D, vol. 75, no. 8, Article ID 084031, 2007.

[13] K. Bamba, C.-Q. Geng, C.-C. Lee, and L.-W. Luo, "Equation of state for dark energy in $f(T)$ gravity," Journal of Cosmology and Astroparticle Physics, vol. 2011, no. 1, article 021, 2011.

[14] K. Bamba, C.-Q. Geng, and C.-C. Lee, “Comment on "einstein's other gravity and the acceleration of the universe," Submitted, 2010, http://arxiv.org/abs/1008.4036vl.

[15] E. Kiritsis and G. Kofinas, "Hořava-Lifshitz cosmology," Nuclear Physics B, vol. 821, no. 3, pp. 467-480, 2009.

[16] T. Nishioka, "Hořava-Lifshitz holography," Classical and Quantum Gravity, vol. 26, no. 24, Article ID 242001, 2009.

[17] R. Myrzakulov, D. Sáez-Gómez, and A. Tureanu, "On the $\Lambda \mathrm{CDM}$ universe in $f(G)$ gravity," General Relativity and Gravitation, vol. 43, no. 6, pp. 1671-1684, 2011.

[18] A. Banijamali, B. Fazlpour, and M. R. Setare, "Energy conditions in $f(G)$ modified gravity with non-minimal coupling to matter," Astrophysics and Space Science, vol. 338, no. 2, pp. 327-332, 2012.

[19] S. Nojiri and S. D. Odintsov, "Modified Gauss-Bonnet theory as gravitational alternative for dark energy," Physics Letters B, vol. 631, no. 1-2, pp. 1-6, 2005.

[20] B. Li, J. D. Barrow, and D. F. Mota, "Cosmology of modified Gauss-Bonnet gravity," Physical Review D, vol. 76, no. 4, Article ID 044027, 2007.

[21] T. P. Sotiriou and V. Faraoni, " $F(R)$ theories of gravity," Reviews of Modern Physics, vol. 82, no. 1, pp. 451-497, 2010.

[22] A. de Felice and S. Tsujikawa, " $f(R)$ theories," Living Reviews in Relativity, vol. 13, no. 3, 2010.

[23] T. P. Sotiriou, " $F(R)$ gravity and scalar-tensor theory," Classical and Quantum Gravity, vol. 23, article 5117, 2006.

[24] S. Capozziello and M. de Laurentis, "Extended theories of gravity," Physics Reports, vol. 509, no. 4-5, pp. 167-321, 2011.

[25] K. Bamba and C.-Q. Geng, "Thermodynamics in $F(R)$ gravity with phantom crossing," Physics Letters B, vol. 679, no. 3, pp. 282-287, 2009.
[26] M. Akbar and R.-G. Cai, "Thermodynamic behavior of field equations for $f(R)$ gravity," Physics Letters $B$, vol. 648, no. 2-3, pp. 243-248, 2007.

[27] N. J. Poplawski, "A Lagrangian description of interacting dark energy," Submitted, 2006, http://arxiv.org/abs/gr-qc/0608031.

[28] T. Harko, F. S. N. Lobo, S. Nojiri, and S. D. Odintsov, " $F(R, T)$ gravity," Physical Review D, vol. 84, no. 2, Article ID 024020, 2011.

[29] R. Myrzakulov, “Dark energy in $F(R, T)$ gravity," Submitted, 2012, http://arxiv.org/abs/1205.5266v2.

[30] M. Jamil, D. Momeni, and R. Myrzakulov, "Attractor solutions in $f(T)$ cosmology," European Physical Journal C, vol. 72, no. 3, pp. 1-10, 2012.

[31] S. Chattopadhyay, "A study on the interacting Ricci dark energy in $f(R, T)$ gravity," Proceedings of the National Academy of Sciences A, 2013.

[32] A. Pasqua, S. Chattopadhyay, and I. Khomenko, "A reconstruction of modified holographic Ricci dark energy in $f(R, T)$ gravity," Canadian Journal of Physics, vol. 91, no. 8, pp. 632-638, 2013.

[33] F. G. Alvarenga, A. de la Cruz-Dombriz, M. J. S. Houndjo, M. E. Rodrigues, and D. Saez-Gomez, "Dynamics of scalar perturbations in $f(R, T)$ gravity," Physical Review D, vol. 87, Article ID 103526, 9 pages, 2013.

[34] S. Chen and J. Jing, "Dark energy model with higher derivative of Hubble parameter," Physics Letters B, vol. 679, no. 2, pp. 144150, 2009.

[35] M. Jamil, E. N. Saridakis, and M. R. Setare, "Thermodynamics of dark energy interacting with dark matter and radiation," Physical Review D, vol. 81, no. 2, Article ID 023007, 2010.

[36] Q. Wu, Y. Gong, A. Wang, and J. S. Alcaniz, "Current constraints on interacting holographic dark energy," Physics Letters B, vol. 659, no. 1-2, pp. 34-39, 2008.

[37] H. Kim, H. W. Lee, and Y. S. Myung, "Equation of state for an interacting holographic dark energy model," Physics Letters B, vol. 632, no. 5-6, pp. 605-609, 2006.

[38] M. R. Setare, "Interacting holographic dark energy model and generalized second law of thermodynamics in a non-flat universe," Journal of Cosmology and Astroparticle Physics, no. 1, article 023, 2007.

[39] B. Wang, Y. Gong, and E. Abdalla, "Transition of the dark energy equation of state in an interacting holographic dark energy model," Physics Letters B, vol. 624, no. 3-4, pp. 141-146, 2005.

[40] K. Karami and A. Sorouri, "Interacting entropy-corrected new agegraphic dark energy in the non-flat universe," Physica Scripta, vol. 82, no. 5, Article ID 025901, 2010.

[41] A. Sheykhi, "Interacting agegraphic tachyon model of dark energy," Physics Letters B, vol. 682, no. 4-5, pp. 329-333, 2010.

[42] V. Sahni, T. D. Saini, A. A. Starobinsky, and U. Alam, "Statefinder-a new geometrical diagnostic of dark energy," Journal of Experimental and Theoretical Physics Letters, vol. 77, no. 5, pp. 201-206, 2003.

[43] U. Alam, V. Sahni, T. D. Saini, and A. A. Starobinsky, "Exploring the expanding universe and dark energy using the statefinder diagnostic," Monthly Notices of the Royal Astronomical Society, vol. 344, no. 4, pp. 1057-1074, 2003.

[44] F. Y. Wang, Z. G. Dai, and S. Qi, "Probing the cosmographic parameters to distinguish between dark energy and modified gravity models," Astronomy and Astrophysics, vol. 507, no. 1, pp. 53-59, 2009. 
[45] P. Wu and H. Yu, "Observational constraints on $f(T)$ theory," Physics Letters B, vol. 693, no. 4, pp. 415-420, 2010.

[46] K. Y. Kim, H. W. Lee, and Y. S. Myung, "Instability of agegraphic dark energy models," Physics Letters B, vol. 660, no. 3, pp. 118124, 2008.

[47] Y. S. Myung, "Instability of holographic dark energy models," Physics Letters B, vol. 652, no. 5-6, pp. 223-227, 2007.

[48] E. Ebrahimi and A. Sheykhi, "Instability of QCD ghost dark energy model," International Journal of Modern Physics D, vol. 20, no. 12, pp. 2369-2381, 2011.

[49] M. Sharif and A. Jawad, "Cosmological evolution of interacting new holographic dark energy in non-flat universe," The European Physical Journal C, vol. 72, article 2097, 2012.

[50] A. Jawad, A. Pasqua, and S. Chattopadhyay, "Correspondence between $f(G)$ gravity and holographic dark energy via powerlaw solution," Astrophysics and Space Science, vol. 344, no. 2, pp. 489-494.

[51] A. Pasqua, S. Chattopadhyay, and I. Khomenko, "On the stability and thermodynamics of the dark energy based on generalized uncertainty principle," International Journal of Theoretical Physics, vol. 52, no. 7, pp. 2496-2507, 2013. 

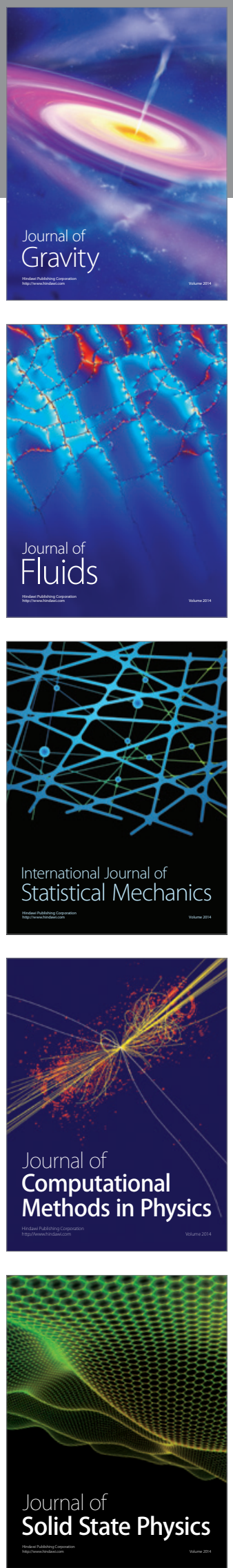

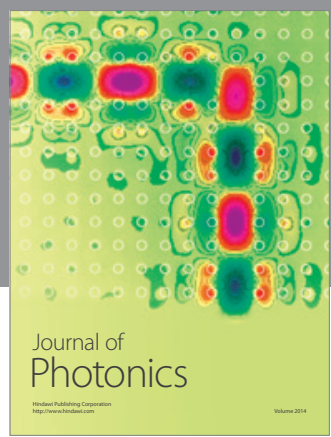

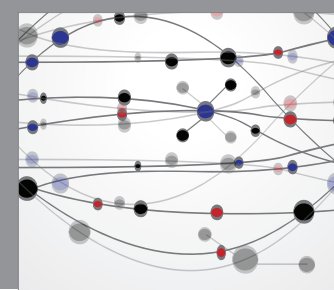

The Scientific World Journal

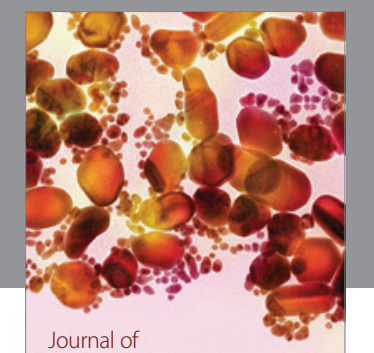

Soft Matter
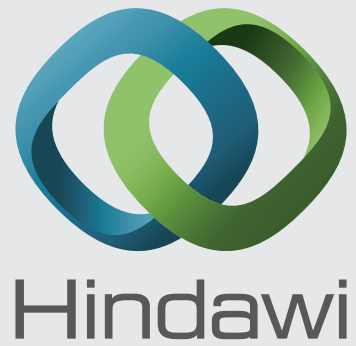

Submit your manuscripts at

http://www.hindawi.com
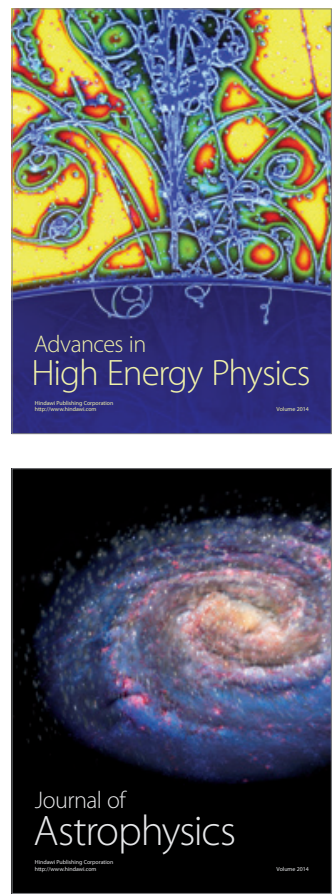
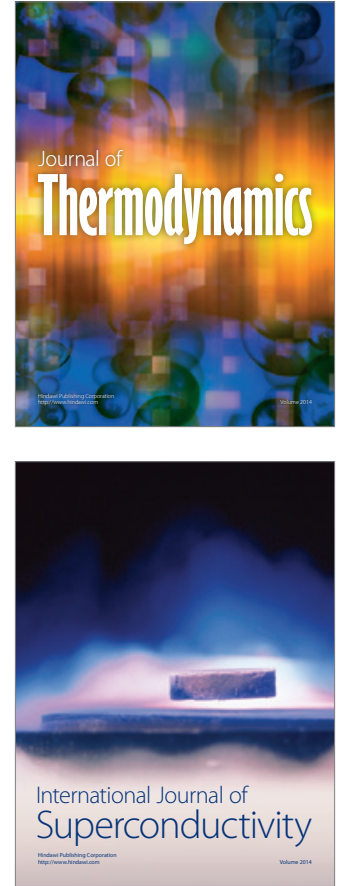
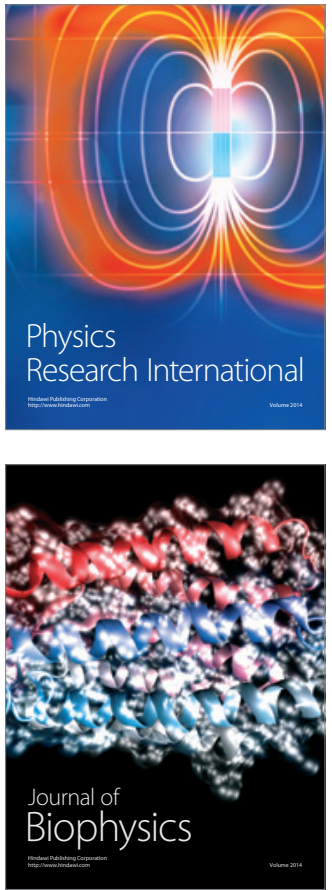
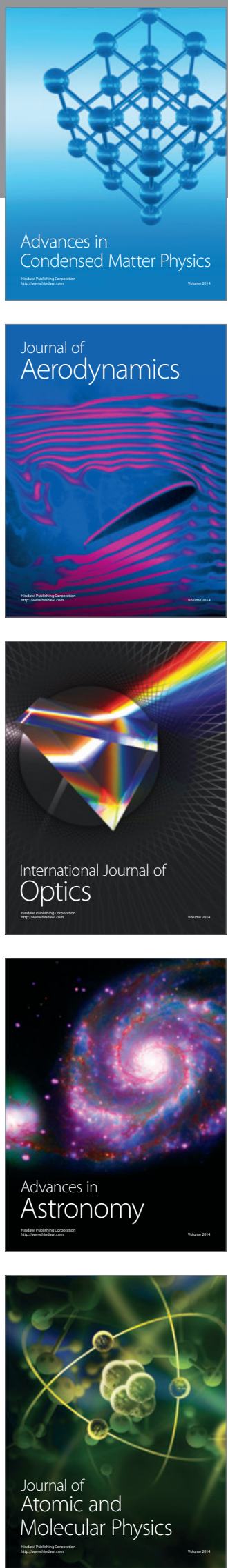